\title{
PHOTOELECTRONIC PROCESSES IN ZnSe:Cu SINGLE CRYSTALS
}

\author{
F. Firszt \\ Institute of Physics, N. Copernicus University \\ Grudziądzka 5/7, 87-100 Toruń, Poland
}

\begin{abstract}
Measurements of steady state and transient stimulation and quenching of photocurrent and luminescence were performed in $\mathrm{ZnSe}$ single crystals doped with $\mathrm{Cu}$ which exhibit marked change in the photoconductivity and luminescence when illuminated by infrared light simultaneously with shorter wavelength radiation. A model which might explain all IR induced phenomena on the basis of donor-acceptor pair recombination mechanism including releasing holes from deep center by IR light and energy redistribution between different recombination centers is presented.
\end{abstract}

PACS numbers: 78.55.Et, 72.40.+w

The $\mathrm{ZnSe}: \mathrm{Cu}$ crystals when excited with $366 \mathrm{~nm}$ UV light exhibit at $35 \mathrm{~K}$ blue $(2.7 \mathrm{eV})$, green $(2.3 \mathrm{eV})$, and red $(1.95 \mathrm{eV})$ luminescence bands due to donor-acceptor (D-A) pairs recombination [1-3]. Green and red emission bands have their characteristic extrinsic excitation spectra with maxima located at $2.7 \mathrm{eV}$ and $2.5 \mathrm{eV}$ and low energy thresholds at $2.55 \mathrm{eV}$ and $2.15 \mathrm{eV}$ respectively. These crystals are photosensitive. The threshold energy of excitation is almost the same for both photoconductivity and red luminescence.

Infrared radiation applied simultaneously with extrinsic excitation causes a significant change in the luminescence and photoconductivity of $\mathrm{ZnSe:Cu}$ crystals. This dynamic transient and steady-state behavior of photoluminescence and photocurrent under illumination with infrared light in addition to shorter wavelength radiation (extrinsic excitation) is shown in Fig. 1.

In order to represent the spectral distribution of steady-state stimulation and quenching by infrared radiation we define the quantity $S$ and $Q$ as the percent of IR induced stimulation for blue and quenching for red luminescence band and photocurrent respectively (see Fig. 1). Employing a specific constant time delays between the beginning of extrinsic and IR illumination and constant time intervals for IR illumination, the spectral dependence of the amplitude of "turn on" $T_{+}$ and "turn off" $T_{-}$transient peaks (see Fig. 1) were measured. Comparing these results with spectral distributions of steady-state stimulation $S$ and quenching $Q$ of luminescence and photoconductivity (Fig. 2), one can see that all of them have the same threshold energy equal to about $0.7 \mathrm{eV}$. 


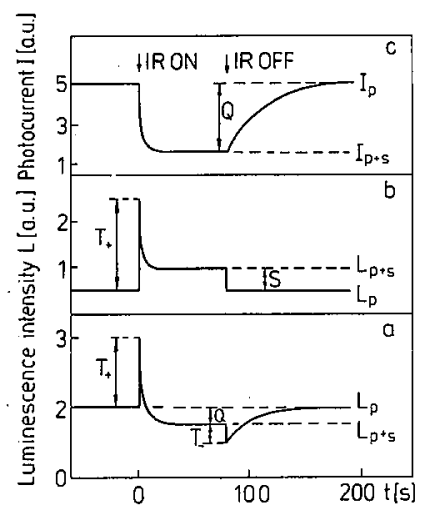

Fig. 1. Typical dynamic behavior showing the influence of IR illumination on the integrated red $a$ and blue $b$ luminescence intensities and photocurrent $c$ for ZnSe:Cu crystals at $T=35 \mathrm{~K}$ under steady-state excitation with light of photon energy $2.5 \mathrm{eV}$.

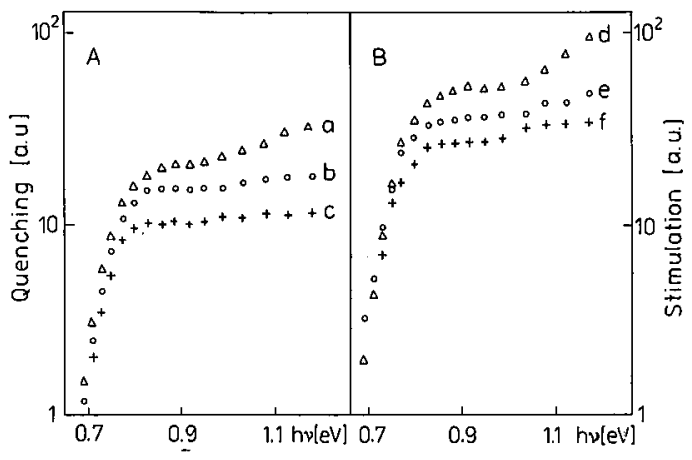

Fig. 2. (A) Spectral dependence of the infrared quenching of steady-state photocurrent $a$, steady-state red emission $b$ and transient $T_{-}$peak (defined in Fig. 1) of red emission $c$; (B) spectral dependence of IR stimulation of transient $T_{+}$peak for blue emission $d$, red emission $e$ and steady-state stimulation of blue emission $f$ in $\mathrm{ZnSe:Cu}$ crystals at $T=35 \mathrm{~K}$.

One of possible explanations of all observed IR induced phenomena can be done assuming a model including the presence, in investigated crystals, of centers responsible for long electron lifetime, i.e. sensitizing centers which have a large capture cross-section for holes, but small for electrons [4-6] with energy level lying about $0.7 \mathrm{eV}$ above the valence band and another centers responsible for radiative and nonradiative recombination processes. The extrinsic excitation with the light of photon energy $2.5 \mathrm{eV}$ produces in $n$-type $\mathrm{ZnSe}: \mathrm{Cu}$ crystals free electrons and bound holes [4] on levels lying about $0.7 \mathrm{eV}$ above the valence band. Under low excitation level the donor acceptor radiative recombination process originates mainly from the distant (slow) D-A pairs [7-9]. At low temperatures infrared light excites electrons from the valence band into hole occupied sensitizing centers. Released 
holes are captured partially by fast luminescence centers (closed D-A pairs) giving rise to an immediate recombination process [10]. The recombination traffic through luminescence centers increases, therefore luminescence is stimulated initially and photoconductivity quenched. This might explain the transient $T_{+}$peak shown in Fig. 1. If the path of recombination was only through red luminescence center, the luminescence would decrease to its original value after the initial rise. Actually, the luminescence intensity of the red band decreases to the value several percent below its original value. At the same time blue luminescence band is only stimulated (Fig. 1b). The quenching takes place when there are different recombination paths in competition which alter the relative rates of recombination. Apart from nonradiative recombination processes the additional recombination path occurs through blue luminescence centers in our crystals. When the infrared radiation is switched off, free holes are no longer created by infrared light in the valence band, therefore they cannot be recaptured by the fast radiative recombination centers. The fast pair recombination ceases and holes are accumulated on slow sensitizing centers, which can explain the initial "turn off" $T_{-}$transient quenching peak of the red emission band (Fig. 1a). The stimulation of the blue luminescence band is stopped at the same time and photocurrent increases to its original value due to hole trapping at sensitizing centers.

Although presented here steady-state and transient stimulation and quenching spectral dependence of photocurrent and photoluminescence have similar threshold energies, it cannot be directly determined from these data that the excitation of red luminescence and photocurrent is from the same center or from different centers with almost the same energy-level position. The observed different thermal quenching behavior of red emission and photoconductivity may indicate that they are different centers as has been already suggested in [11, 12].

\section{References}

[1] S. Iida, J. Phys. Soc. Jpn. 26, 1140 (1969).

[2] M. Godlewski, W.E. Lamb, B.C. Cavenett, Solid State Commun. 39, 595 (1981).

[3] G.B. Stringfellow, R.H. Bube, Phys. Rev. 171, 903 (1968).

[4] H.G. Grimmeiss, C. Oven, R. Mach, J. Appl. Phys. 50, 6328 (1979).

[5] H,G. Grimmeiss, C. Oven, W. Ludwig, R. Mach, J. Appl. Phys. 48, 5122 (1977).

[6] H.G. Grimmeiss, C. Oven, J.W. Allen, J. Appl. Phys. 47, 1103 (1976).

[7] D.G. Thomas, J.J. Hopfield, W.M. Augustyniak, Phys. Rev. 140, A202 (1965).

[8] D.G. Thomas, M. Gersherson, F.A. Trumbore, Phys. Rev. 133, A269 (1964).

[9] W.E. Hagston, J. Lumin. 3, 253 (1971); 5, 285 (1972).

[10] H.G. Grimmeiss, B. Monemar, Phys. Status Solidi A 19, 505 (1973).

[11] Jia-zhen Zheng, J.M. Allen, H.M. Yates, J.O. Williams, J. Cryst. Growth 117, 358 (1992).

[12] Jia-zhen Zheng, J.W. Allen, in: Proc. Int. Conf. Optical Characterization Semicond., Sofia 1990, to be published. 